01

\title{
Определение отношения Mn/Fe в железомарганцевых конкрециях с помощью безэталонной лазерно-искровой эмиссионной спектроскопии
}

\author{
(C) Т.Ф. Ахметжанов ${ }^{1}$, Т.А. Лабутин ${ }^{1}$, С.М. Зайцев ${ }^{1}$, А.Н. Дроздова ${ }^{2}$, А.М. Попов ${ }^{1, \text { ฯ }}$ \\ ${ }^{1}$ Московский государственный университет имени М.В. Ломоносова, химический фракультет, \\ 119234 Москва, Россия \\ ${ }^{2}$ Институт океанологии имени П.П. Ширшова РАН, \\ 117997 Москва, Россия \\ ฯ e-mail: popov@laser.chem.msu.ru
}

Поступила в редакцию 24.10.2018 г.

В окончательной редакции 14.12.2018 г.

Принята к публикации 18.12.2018 г.

\begin{abstract}
Для определения отношения $\mathrm{Mn} / \mathrm{Fe}$ в железомарганцевых конкрециях применен метод безэталонной лазерно-искровой эмиссионной спектроскопии, основанный на использовании только температуры и электронной плотности плазмы и интенсивностей аналитических линий марганца и железа (Mn I 447.28 и $447.93 \mathrm{~nm}$, Fe I $447.65 \mathrm{~nm}$ ). Температура плазмы была определена по методу двух линий (Mn I 447.28 и $447.93 \mathrm{~nm}$ ), а ее электронная плотность - по штарковскому уширению линии $\mathrm{Mg}$ I $517.26 \mathrm{~nm}$. Для исследования использовался как набор стандартных образцов состава (серия ООПЕ), так и образцы железомарганцевых конкреций, отобранные в ходе экспедиций в Карское море и море Лаптевых. Продемонстрирована возможность полуколичественного определения отношения $\mathrm{Mn} / \mathrm{Fe}$ в диапазоне значений от 0.2 до 4.2, что достаточно для определения данного отношения в большинстве типов конкреций различного географического происхождения. Данные безэталонного анализа коррелируют с результатами атомно-эмиссионного анализа с индуктивно-связанной плазмой.
\end{abstract}

DOI: $10.21883 /$ OS.2019.04.47506.310-18

\section{Введение}

Лазерно-искровая эмиссионная спектрометрия (ЛИЭС) является одним из наиболее перспективных методов прямого экспресс-анализа твердых, жидких и газообразных образцов [1]: металлов [2], включая легкие сплавы [3], метеоритов [4], почв и руд [5,6], растительных образцов [7]. Применение экспрессных методов элементного анализа исключительно актуально для оптимизации процедуры поиска и отбора проб пелагических отложений во время морских экспедиций. Помимо этого, мониторинг содержания токсичных элементов ( $\mathrm{Pb}, \mathrm{Hg}, \mathrm{Cu}, \mathrm{Zn}$ и др.) в акваториях арктических морей необходим для осуществления экологического контроля [8]. Принципиальная возможность проведения ЛИЭС-анализа донных отложений на борту корабля продемонстрирована во время XVI итальянской экспедиции в Антарктике [9]. Однако неоднородность железомарганцевых конкреций (ЖМК) [10] исключает применение традиционного градуировочного графика из-за влияния матричных эффектов на параметры лазерной плазмы. Поскольку ЛИЭС позволяет проводить многоэлементный анализ без использования стандартных образцов сравнения (безэталонная ЛИЭС) $[11,12]$, использование подобного подхода при анализе ЖМК предпочтительно. Отношение $\mathrm{Mn} / \mathrm{Fe}$ является маркером содержания в ЖМК редкоземельных и ряда других цветных металлов, что позволяет судить о „ценности“ конкреций, отобранных в каждом отдельно взятом регионе. Таким образом, задачей нашей работы было исследование возможности определения отношения $\mathrm{Mn} / \mathrm{Fe}$ в образцах ЖМК методом безэталонной ЛИЭС.

\section{Эксперимент}

Измерения проводились на экспериментальной установке (рис. 1), подробное описание которой дано в [13]. Излучение второй гармоники $\mathrm{Nd}$ :АИГ-лазера с лампо-

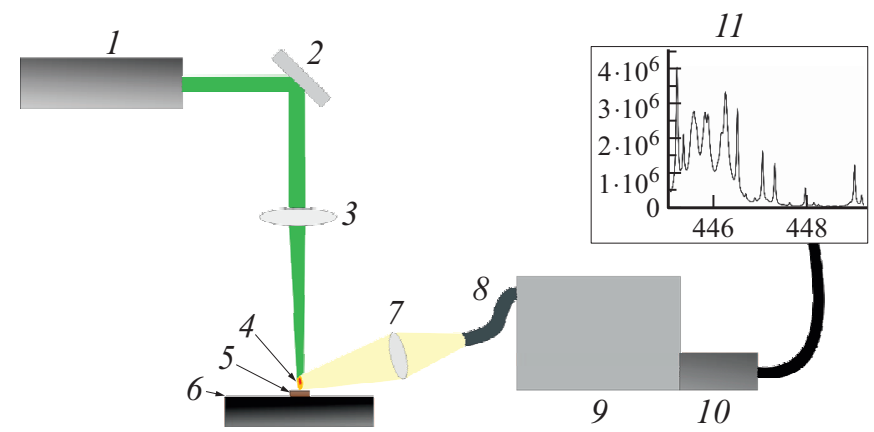

Рис. 1. Схематическое представление установки ЛИЭС: 1 лазер, 2 - система зеркал, 3, 7 - собирающие линзы, 4 плазма, 5 - образец, 6 - подложка, 8 - оптоволокно, 9 спектрограф, 10 - детектор, 11 - система вывода. 
Таблица 1. Состав стандартных образцов пелагических осадков (wt.\%)

\begin{tabular}{c|c|c|c|c}
\hline $\begin{array}{c}\text { Индекс } \\
\text { образца }\end{array}$ & ООПЕ 601 & ООПЕ 602 & ООПЕ 603 & ООПЕ 604 \\
\hline $\begin{array}{c}\text { Тип пела- } \\
\text { гического } \\
\text { осадка }\end{array}$ & ЖМК & ЖМК & ЖМК & $\begin{array}{c}\text { корка } \\
\text { рудная }\end{array}$ \\
\hline $\mathrm{Mn}$ & $23.0 \pm 0.2$ & $27.0 \pm 0.2$ & $19.4 \pm 0.2$ & $15.3 \pm 0.2$ \\
\hline $\mathrm{Fe}$ & $12.1 \pm 0.1$ & $6.50 \pm 0.05$ & $17.4 \pm 0.2$ & $15.5 \pm 0.1$
\end{tabular}

вой накачкой „LOTIS LS-2134 UTF“ $(532 \mathrm{~nm}$, энергия импульса $80.1 \mathrm{~mJ}$, длительность $6 \mathrm{ns,} \mathrm{частота} 5 \mathrm{~Hz}$ ) фокусировали на поверхность образца с помощью ахроматического дублета с $f=150 \mathrm{~mm}$. Излучение лазерноиндуцированной плазмы с помощью асферической кварцевой линзы с $f=30 \mathrm{~mm}$ собирали на торец оптоволокна линия-линия. Спектры регистрировали с помощью широкоапертурного $(f / 4)$ спектрометра типа ЧерниТернера „HR 320“ (ISA, CШA), оснащенного ПЗCкамерой с усилителем яркости. Дифракционная решетка $36001 / \mathrm{mm}$ и входная щель шириной $25 \mu \mathrm{m}$ обеспечивали высокое спектральное разрешение $\Delta \lambda=0.02-0.12 \AA$ в диапазоне $440-520 \mathrm{~nm}$. Для увеличения отношения сигнал/шум накопление эмиссионных спектров проводили по 10 импульсам. Анализируемый образец перемещали в горизонтальном направлении с помощью трансляционного столика, чтобы уменьшить вариации эмиссионного сигнала от импульса к импульсу. Кроме того, для снижения влияния неоднородности проб проводилось 20 параллельных измерений. Отнесение линий проводили на основании разработанного нами алгоритма [14] с использованием базы спектральных данных NIST [15]. Этот алгоритм основан на сравнении спектра, моделируемого для определенных параметров плазмы (температура и электронная плотность), и экспериментального, и позволяет найти линии определяемых элементов с минимальными спектральными помехами со стороны других линий. Контуры спектральных линий за вычетом непрерывного фона - сплошного спектра испускания плазмы - аппроксимировали лоренцевым профилем с помощью программного пакета OriginPro 8.1. Интенсивностью линии служила площадь контура линии, в то время как полная ширина на полувысоте принималась за ширину для расчета электронной плотности.

\section{Образцы}

Двенадцать образцов ЖМК были отобраны в 22 точках сбора со дна Карского моря и моря Лаптевых в ходе двух арктических экспедиций: 129-го рейса НИС „Профессор Штокман“ (2014, ПШ-129) и 63-го рейса
Таблица 2. Идентифицированные линии, наблюдаемые в спектрах конкреций

\begin{tabular}{c|c|c|c|c}
\hline$\lambda, \mathrm{nm}$ & Element & $E, \mathrm{eV}$ & $g$ & $A, \mathrm{~s}^{-1}$ \\
\hline 446.47 & Mn I & 5.696 & 6 & $4.39 \cdot 10^{7}$ \\
\hline 446.58 & Ti I & 4.515 & 7 & $3.28 \cdot 10^{7}$ \\
\hline 446.66 & Fe I & 5.607 & 7 & $1.20 \cdot 10^{7}$ \\
\hline 446.85 & Ti II & 3.905 & 8 & $1.0 \cdot 10^{7}$ \\
\hline 446.94 & Fe I & 6.427 & 7 & $1.59 \cdot 10^{7}$ \\
\hline 447.02 & Mn I & 5.714 & 4 & $3.00 \cdot 10^{7}$ \\
\hline 447.12 & Ti I & 4.506 & 5 & $5.26 \cdot 10^{7}$ \\
\hline 447.28 & Mn I & 5.724 & 2 & $4.35 \cdot 10^{7}$ \\
\hline 447.48 & Ti I & 4.873 & 9 & $9.77 \cdot 10^{5}$ \\
& Ti I & 4.213 & 5 & $9.77 \cdot 10^{5}$ \\
\hline 447.60 & Fe I & 5.615 & 5 & $1.01 \cdot 10^{7}$ \\
& Fe I & 6.456 & 5 & $3.39 \cdot 10^{7}$ \\
\hline 447.93 & Mn I & 7.879 & 10 & $3.40 \cdot 10^{7}$ \\
\hline 447.97 & Ti I & 4.501 & 3 & $2.95 \cdot 10^{7}$ \\
\hline 517.16 & Fe I & 3.882 & 9 & $4.46 \cdot 10^{5}$ \\
\hline 517.29 & Mg I & 5.108 & 3 & $3.37 \cdot 10^{7}$ \\
\hline 517.37 & Ti I & 2.396 & 5 & $4.23 \cdot 10^{6}$
\end{tabular}

НИС „Академик Мстислав Келдыш“ (2015, АМК-63). Образцы дисковидной и чашевидной форм были извлечены с глубин от $19 \mathrm{~m}$ на станции 35 , расположенной к западу от полуострова Ямал, до $230 \mathrm{~m}$ во впадине Воронина (станция 5239). Диаметр конкреций варьировал от 2 до $14 \mathrm{~cm}$. Цвет ЖМК варьировал от светлооранжевого, типичного для слоев оксидов железа, до черного, обусловленного присутствием оксигидроксидов марганца. Было установлено, что цвет ЖМК не зависит от размера и формы конкреции подобно образцам, отобранным из северо-восточной части Карского моря [16]. Образцы высушивали при $60^{\circ} \mathrm{C}$, истирали в агатовой ступке, просеивали через сито с размером ячейки $50 \mu \mathrm{m}$ и хранили в полиэтиленовых пакетах для дальнейшего анализа. Содержание железа и марганца в образцах ЖМК было определено с помощью атомно-эмиссионной спектрометрии с индуктивно-связанной плазмой (АЭС-ИСП, іСАР-6500, Thermo Scientific, США) после разложения $100 \mathrm{mg}$ образца в смеси четырех кислот. Кроме того, использовался ряд стандартных образцов комплекта ООПЕ - осадочные отложения пелагические - наиболее близких по составу и физическим характеристикам к отобранным пробам. Содержание железа и марганца в образцах ООПЕ приведено в табл. 1 согласно паспортным данным. Средний размер частиц в порошкообразном материале стандартных 

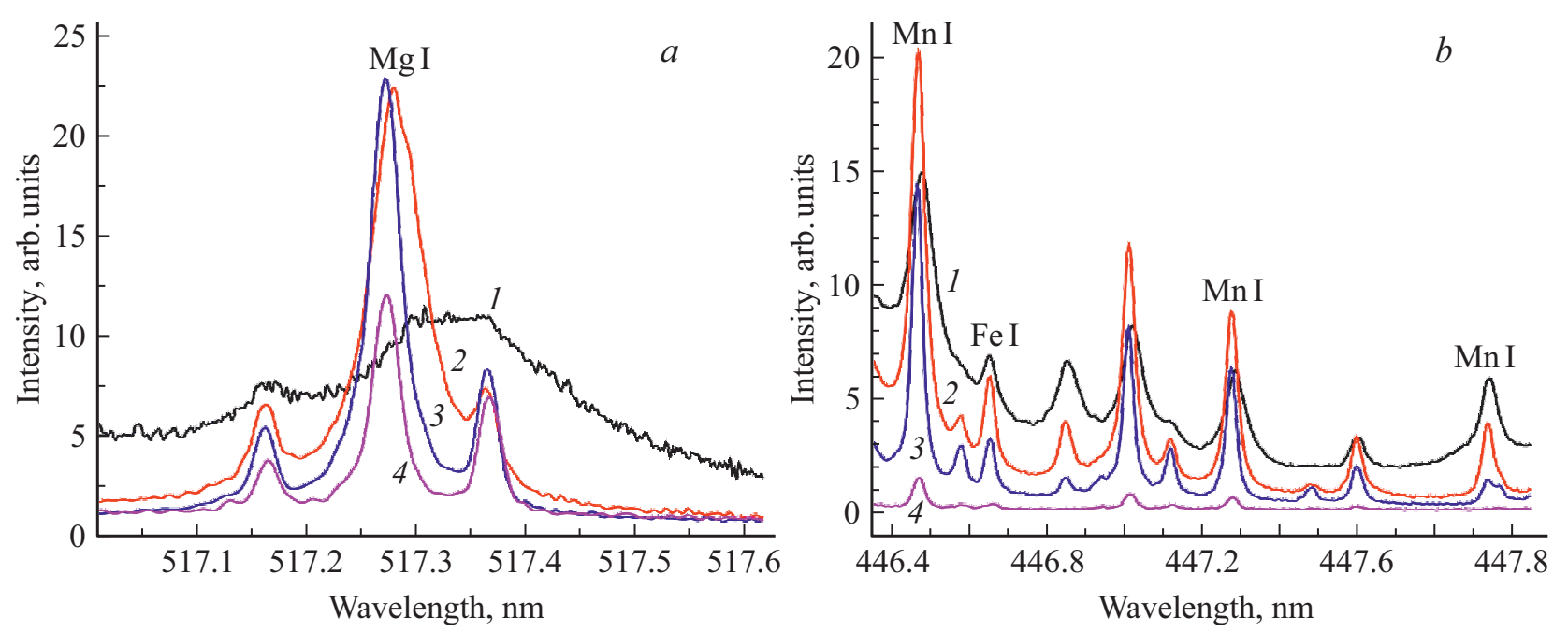

Рис. 2. Эмиссионные спектры лазерной плазмы для образца ООПЕ 603 в области линии $\mathrm{Mg}$ I $517.26 \mathrm{~nm}(a)$ и в области аналитических линий железа и марганца $(b)$ для разных времен задержки: $1(1), 2.5(2), 5(3), 10 \mu \mathrm{s}(4)$.

образцов варьировал от 30 до $90 \mu \mathrm{m}$. Все образцы ЖМК и ООПЕ были спрессованы в таблетки диаметром $15 \mathrm{~mm}$ с помощью гидравлического пресса при давлении $5 \mathrm{mpa}$.

\section{Диагностика плазмы}

Линии, идентифицированные в спектрах конкреций (диапазоны $446.4-448$ и $517.0-517.8 \mathrm{~nm}$ ), приведены в табл. 2. Электронную плотность определяли по штарковскому уширению линии $\mathrm{Mg}$ I $517.26 \mathrm{~nm}$, электронный ударный параметр которой известен с хорошей точностью $-6.56 \mathrm{pm}$ при $n_{e}=10^{16} \mathrm{~cm}^{-3}$ и $T=10^{4} \mathrm{~K}$ [17]. На рис. 2, $a$ демонстрируется эволюция профиля линии Mg I $517.26 \mathrm{~nm}$ для разных времен задержки. Как видно, для коротких задержек контур линии имеет большую ширину и смещен в красную область, что согласуется с нашими предыдущими исследованиями при испарении алюминиевых сплавов [18]. Как видно из рис. $2, a$, наблюдаемая ширина линии магния составляет более $50 \mathrm{pm}$, поэтому допплеровской $\left(\sim 0.4 \mathrm{pm}\right.$ при $\left.T=10^{4} \mathrm{~K}\right)$ и инструментальной $(2.5 \mathrm{pm}$ при $517 \mathrm{~nm})$ ширинами контура линии мы пренебрегали. Отсутствие заметной асимметрии контура линии магния позволяет использовать ее для определения электронной плотности плазмы. Для квадратичного эффекта Штарка полная ширина на полувысоте определяется [17] из уравнения

$$
w\left(n_{e}, T\right)=2 w_{s}(T)\left[1+g A(T) 10 .^{-4}\right] n_{e} 10^{-16},
$$

где $g=1.75(1-0.75 a), n_{e}-$ электронная плотность, $w_{s}$ - электронный ударный параметр, $A(T)$ - параметр ионного уширения, $a$ - дебаевский радиус. Пренебрегая дебаевским экранированием положительных ионов, (1) можно представить в виде

$$
n_{e}=\frac{w\left(n_{e}, T\right)}{2 w_{s}(T) 10^{-16}} .
$$

Температуру плазмы определяли по уравнению

$$
T=\frac{E_{2}-E_{1}}{k}\left(\ln \frac{A_{2} g_{2} I_{1} \lambda_{1}}{A_{1} g_{1} I_{2} \lambda_{2}}\right)^{-1}
$$

где $E, A, g, I, \lambda$ - энергия верхнего уровня, коэффициент Эйнштейна, статистический вес верхнего уровня, интенсивность линии и ее длина волны соответственно. Для этого использовали две линии марганца Mn I 447.28 и $447.93 \mathrm{~nm}$, параметры которых приведены в табл. 2 в соответствии с данными из базы атомных линий NIST [15]. Данные линии Mn I (447.28 и $447.93 \mathrm{~nm})$ были выбраны нами в соответствии со следующими соображениями. Во-первых, как видно из рис. $2, b$ эти линии достаточно интенсивны, т.е. погрешность, вносимая фоном, пренебрежимо мала. Во-вторых, для максимального уменьшения погрешности эксперимента мы выбрали линии с наибольшей разницей энергий верхних уровней $(2.155 \mathrm{eV})$.

На рис. 3 сравниваются эволюция температуры плазмы (кривая 1) и ее электронная плотность (кривая 2). Как видно, оба параметра уменьшаются экспоненциально, причем электронная плотность уменьшается в $\sim 10$ раз, в то время как температура плазмы в $\sim 2$ раза. Полученные нами значения $T$ и $N_{e}$ в пределах погрешности согласуются с литературными данными по лазерноиндуцированному испарению почв $[19,20]$. Это может косвенно подтвердить отсутствие самопоглощения и обоснованность выбора данных линий для расчетов. Для проверки выполнения необходимых условий для существования локального термодинамического равновесия 


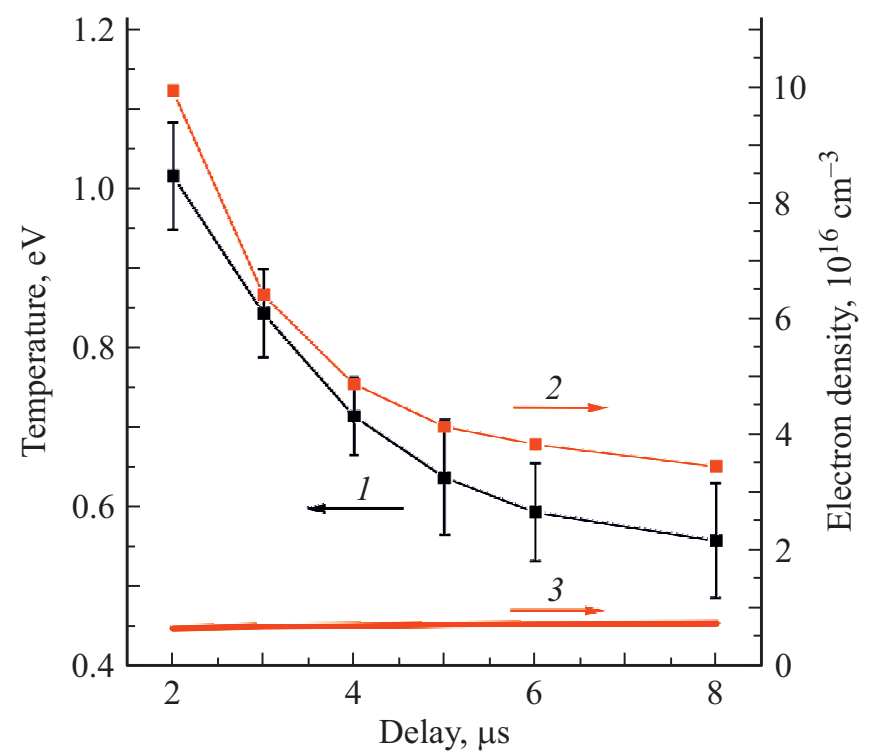

Рис. 3. Эволюция температуры (1, левая шкала), электронной плотности (2, правая шкала) и критического значения электронной плотности плазмы по критерию МакУиртера-Хея (3, правая шкала) для образца ООПЕ 603.

(ЛТР) в лазерной плазме мы использовали критерий МакУиртера-Хэя [21]

$$
n_{e}>\frac{2.55 \cdot 10^{11}}{\langle\bar{g}\rangle} T^{1 / 2}(\Delta E)^{3} \text {, }
$$

где $\Delta E-$ разность между верхним и нижним уровнями линии $\mathrm{Mg}$ I $517.26 \mathrm{~nm}$, а $\langle\bar{g}\rangle$ - безразмерный фактор Гаунта. Как видно из рис. 3 (кривая 3), значения электронной плотности сильно превосходят критические значения по указанному критерию.

\section{Результаты}

Основной проблемой применения ЛИЭС для количественного анализа сложных объектов являются матричные эффекты, которые проявляются значительными вариациями параметров плазмы от образца к образцу. На рис. 4 представлена температура (кривая 1) и электронная плотность (кривая 2) плазмы для всех образцов: если температура варьирует незначительно (в пределах $0.85-0.95 \mathrm{eV}$ ), то электронная плотность меняется существенно сильнее. Такого рода эффекты оказывают значительное влияние при построении градуировочной зависимости, что приводит в конечном счете к большой систематической погрешности аналитических измерений. Недавно похожий эффект наблюдался для почв и руд [22]. Тем не менее отметим, что величина электронной плотности оказывает меньшее влияние на результаты безэталонного анализа, чем значения температуры $[1,12]$.
Одним из способов нивелирования матричных эффектов являются методы безэталонного ЛИЭС. Интенсивность эмиссионной линии $I$ можно записать следующим образом [11]:

$$
I=\alpha \frac{h c}{\lambda} \frac{N}{Z(T)} g_{j} A \exp \left(-E_{j} / k T\right),
$$

где $\alpha$ - экспериментальный параметр, учитывающий эффективность сбора излучения, $N$ - число частиц, $Z(T)$ - сумма по состояниям, $g_{j}$ и $E_{j}-$ статистический вес и энергия верхнего уровня для данного перехода, $h, k$ и $c$ - постоянные Планка, Больцмана и скорость света соответственно. Для учета ионизации атомов в плазме соотношение атомов (верхний индекс $I$ ) и ионов

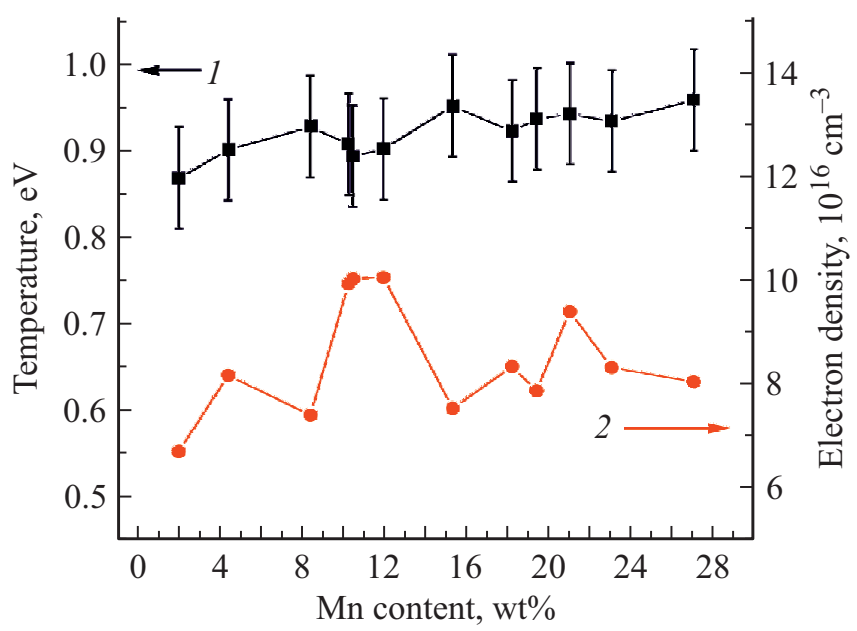

Рис. 4. Зависимость температуры (1, левая шкала) и электронной плотности (2, правая шкала) плазмы от содержания марганца в образцах конкреций.

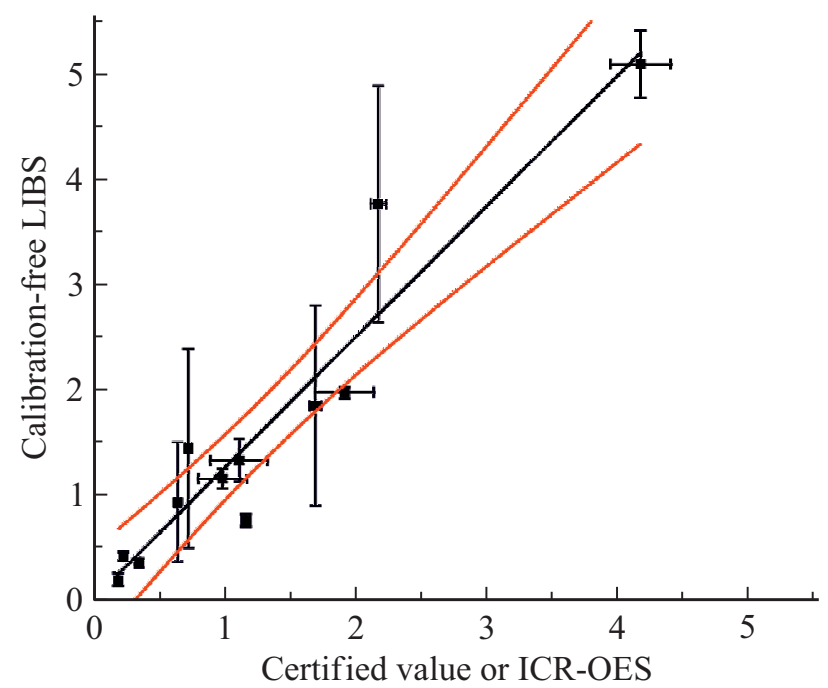

Рис. 5. Сравнение результатов определения отношения $\mathrm{Mn} / \mathrm{Fe}$, полученных с помощью безэталонного варианта ЛИЭС и ИСП-АЭС или сертифицированными значениями. Границы доверительного интервала $(P=0.95)$ обозначены красными линиями. $R^{2}=0.907$. 
(верхний индекс II) можно определить из уравнения Caxa [11]:

$$
\frac{N^{I I}}{N^{I}}=\frac{1}{n_{e}} 2 \frac{Z^{I I}(T)}{Z^{I}(T)}\left(\frac{m_{e} k T}{2 \pi \hbar^{2}}\right)^{3 / 2} \exp \left(-\frac{E_{\infty}}{k T}\right),
$$

где $m_{e}-$ масса электрона и $E_{\infty}-$ потенциал ионизации. Отношение массовых долей железа и марганца в образце будет тогда определяться исходя из отношения интенсивностей атомных линий следующим выражением:

$$
\begin{aligned}
\frac{\omega_{\mathrm{Mn}}}{\omega_{\mathrm{Fe}}}= & \frac{M_{\mathrm{Mn}} I_{\mathrm{Mn}} Z_{\mathrm{Mn}}^{I}(T)}{M_{\mathrm{Fe}} I_{\mathrm{Fe}} Z_{\mathrm{Fe}}^{I}(T)} \frac{g_{\mathrm{Fe}} A_{\mathrm{Fe}}}{g_{\mathrm{Mn}} A_{\mathrm{Mn}}} \\
& \times \exp \left(\frac{E_{\mathrm{Mn}}-E_{\mathrm{Fe}}}{k T}\right) \frac{\left(1+\frac{N_{\mathrm{Mn}}^{I I}}{N_{\mathrm{Mn}}^{I}}\right)}{\left(1+\frac{N_{\mathrm{Fe}}^{I I}}{N_{\mathrm{Fe}}^{I}}\right)},
\end{aligned}
$$

а отношения ионов к атомам определяется по уравнению (6).

Используя полученные значения температуры и электронной плотности для $t=2.5 \mu \mathrm{s}$ для каждого образца, мы определили отношение $\mathrm{Mn} / \mathrm{Fe}$ в 12 образцах ЖМК по уравнению (7). На рис. 5 сравниваются полученные значения с данными АЭС-ИСП-анализа и паспортными содержаниями железа и марганца в стандартных образцах. Линейная аппроксимация

$$
a c_{\text {АЭС-ИСП }}+b=c_{\text {CF-LIBS }}
$$

имеет высокий коэффициент линейной корреляции $\left(R^{2}=0.907\right)$ с полным отсутствием аддитивной систематической погрешности $(b=0.0 \pm 0.1)$. Тем не менее четыре образца имеют высокие погрешности определения отношения $\mathrm{Mn} / \mathrm{Fe}(>30 \%)$. Причиной этого может быть в первую очередь неоднородная слоистая структура некоторых конкреций. Поскольку тангенс угла наклона прямой отклоняется от единицы $(a=1.25 \pm 0.1)$, можно говорить о наличии мультипликативной погрешности, которая, вероятнее всего, вызвана сильными спектральными помехами линий Mn I 446.22 и $446.47 \mathrm{~nm}$, на крыле которых находится аналитическая линия железа, как видно из рис. 2.

\section{Выводы}

Лазерно-искровая эмиссионная спектрометрия позволяет проводить полуколичественное определение отношения основных элементов ( $\mathrm{Mn}, \mathrm{Fe})$ в ЖМК без построения градуировочного графика в диапазоне содержаний марганца 1.92-27.02 wt.\% и железа 6.5-23.52 wt.\%. Среди основных проблем можно выделить как традиционные в эмиссионной спектроскопии спектральные интерференции, так и неоднородность слоистой структуры конкреций. Значения отношения $\mathrm{Mn} / \mathrm{Fe}$ в ЖМК, полученные безэталонным вариантом ЛИЭС, оказались несколько завышенными по сравнению с данными традиционного химического анализа АЭС-ИСП. Учитывая коэффициент пропорциональности между результатами двух методов $(1: 1.25)$, можно утверждать, что безэталонный вариант ЛИЭС применим для экспрессной классификации ЖМК.

Работа выполнена в рамках государственного задания № 0149-2018-0005 (в части химического анализа ЖМК с помощью АЭС-ИСП) и соглашения № 18-13-00269 с Российским научным фондом (в части диагностики лазерной плазмы).

\section{Список литературы}

[1] Hahn D.W., Omenetto N. // Appl. Spectrosc. 2012. V. 66. P. 347. doi 10.1366/11-06574

[2] Noll R. Laser-induced breakdown spectroscopy: Fundamentals and Applications. Springer-Verlag, 2012. 489 p.

[3] Lednev V.N., Yakovlev A.V., Labutin T.A. et al. // J. Anal. Chem. 2007. V. 62. P. 1151. doi 10.1134/S106193480712009X

[4] De Giacomo A., Dell'Aglio M., De Pascale O. et al. // Spectrochim. Acta. B. 2007. V. 62. P. 1606. doi 10.1016/j.sab.2007.10.004

[5] Лабутин Т.А., Попов А.М., Зайцев С.М., Калько И.А., Зоров Н.Б. // Опт. и спектр. 2016. Т. 121. С. 367; Labutin T.A., Popov A.M., Zaytsev S.M. Cal'ko I.A., Zorov N.B. // Opt. Spectrosc. 2016. V. 121. P. 339. doi 10.1134/S0030400X16080129

[6] Popov A.M., Labutin T.A., Zaytsev S.M. et al. // J. Anal. At. Spectrom. 2014. V. 29. P. 1925. doi 10.1039/c4ja00199k

[7] Nunes L.C., Braga J.W.B., Trevizan L.C. et al. // J. Anal. At. Spectrom. 2010. V. 25. P. 1453. doi 10.1039/C003620J

[8] Демина Л.Л. Формы миграции тяжелых металлов в океане (на ранних стадиях океанского осадкообразования). М.: Наука, 1982. $120 \mathrm{c}$.

[9] Barbini R., Colao F., Lazic V. et al. // Spectrochim. Acta. B. 2002. V. 57. P. 1203. doi 10.1016/S0584-8547(02)00055-1

[10] Kolesnik O.N., Kolesnik A.N. // Russ. Geol. Geophys. 2013. V. 54. P. 653. doi 10.1016/j.rgg.2013.06.001

[11] Ciucci A., Corsi M., Palleschi V. et al. // Appl. Spectrosc. 1999. V. 53. P. 960. doi 10.1366/0003702991947612

[12] Tognoni E., Cristoforetti G., Legnaioli S. et al. // Spectrochim. Acta. B. 2010. V. 65. P. 1. doi 10.1016/j.sab.2009.11.006

[13] Zaytsev S.M., Popov A.M., Zorov N.B. et al. // J. Instrum. 2014. V. 9. N P06010. doi 10.1088/1748-0221/9/06/P06010

[14] Labutin T.A., Zaytsev S.M., Popov A.M. // Anal. Chem. 2013. V. 85. P. 1985. doi 10.1021/ac303270q

[15] Kramida A., Ralchenko Y., Reader J. NIST ASD Team. NIST Atomic Spectra Database (ver. 5.3). Электронный ресурс. Режим доступа: http://physics.nist.gov/asd

[16] Shulga N.A., Drozdova A.N., Peresypkin V.I. // Dokl. Earth Sci. 2017. V. 472. P. 237. doi 10.1134/S1028334X17020246

[17] Грим Г. Уширение спектральных линий в плазме. М.: Мир, 1978. $491 \mathrm{c.}$

[18] Попов А.М., Лабутин Т.А., Зайщев С.М., Зоров Н.Б. // Опт. и спектр. 2017. Т. 123. С. 503; Popov A.M., Labutin T.A., Zaytsev S.M., Zorov N.B. // Opt. Spectrosc. 2017. V. 123. P. 521. doi 10.1134/S0030400X17100216 
[19] Eppler A.S., Cremers D.A., Hickmott D.D. et al. // Appl. Spectrosc. 1996. V. 50. P. 1175. doi $10.1366 / 0003702963905123$

[20] Multari R.A., Foster L.E., Cremers D.A. et al. // Appl. Spectrosc. 1996. V. 50. P. 1483. doi 10.1366/0003702963904593

[21] Cristoforetti G., De Giacomo A., Dell'Aglio M. et al. // Spectrochim. Acta. B. 2010. V. 65. P. 86. doi 10.1016/j.sab.2009.11.005

[22] Popov A.M., Zaytsev S.M., Seliverstova I.V. et al. // Spectrochim. Acta. B. 2018. V. 148. P. 205. doi $10.1016 /$ j.sab.2018.07.00 